\title{
Relationship between maternal vitamin $D$ status in the first trimester of pregnancy and maternal and neonatal outcomes: a retrospective single center study
}

Meng $\mathrm{Ni}^{1,2,3}$, Qianqian Zhang ${ }^{1,2,3}$, Jiuru Zhao ${ }^{1,2,3}$, Qianwen Shen ${ }^{1,2,3}$, Dongting Yao ${ }^{1,2,3}$, Tao Wang ${ }^{1,2,3}$ and Zhiwei Liu ${ }^{1,2,3^{*}}$

\begin{abstract}
Background: This study aimed to investigate the relationship between maternal serum vitamin D status in the first trimester of pregnancy and maternal as well as neonatal outcomes, considered the prevalence of vitamin $D$ deficiency (serum $25(\mathrm{OH}) \mathrm{D}<50 \mathrm{nmol} / \mathrm{L}$ ) around the world, especially in the pregnant women.

Methods: From January 2015 to December 2016, in this cross-sectional retrospective study, we enrolled women receiving regular prenatal examinations and giving birth in the International Peace Maternity and Child Health Hospital. Cases confirmed as multiple pregnancy, incomplete medical records, and vitamin D level recorded after 13 weeks of gestation were excluded. A total of 23,394 mother-infant pairs were included ultimately. Obstetric and neonatal information were extracted from the database. Maternal serum vitamin D concentration was measured by chemiluminescence microparticle immunoassay. Logistic regression analysis (unadjusted and adjusted models) was used to analyze the association between vitamin $\mathrm{D}$ and maternal and neonatal outcomes.
\end{abstract}

Results: The average $25(\mathrm{OH}) \mathrm{D}$ concentration was $43.20 \pm 0.10 \mathrm{nmol} / \mathrm{L} ; 67.09 \%$ of patients were vitamin $\mathrm{D}$ deficient $(25(\mathrm{OH}) \mathrm{D}<50.00 \mathrm{nmol} / \mathrm{L}), 29.84 \%$ were vitamin $\mathrm{D}$ insufficient $(50 \mathrm{nmol} / \mathrm{L} \leq 25(\mathrm{OH}) \mathrm{D}<75 \mathrm{nmol} / \mathrm{L}), 3.07 \%$ were sufficient $(25(\mathrm{OH}) \mathrm{D} \geq 75 \mathrm{nmol} / \mathrm{L})$. The maternal $25(\mathrm{OH}) \mathrm{D}$ levels varied with age, pre-pregnancy BMl, season when blood sample was collected, number of previous-pregnancy. Notably, newborns delivered by women with deficient vitamin D status had a higher incidence rate of admission to NICU (Deficiency: 12.20\% vs Insufficiency: $10.90 \%$ vs Sufficiency: $11.70 \%, P_{\text {bonferroni }}=.002$ ) and a longer stay (deficiency: $6.2 \pm 4.1$ days vs insufficiency: $5.9 \pm 3.1$ days vs sufficiency: $5.1 \pm 2.1$ days, $P_{\text {bonferroni }}=.010$ ). Moreover, maternal vitamin $D$ deficiency was a dependent risk factor for admission to NICU (unadjusted $\mathrm{OR}=1.35,95 \% \mathrm{Cl}, 1.05-1.74 P_{\text {bonferroni }}=.022$; adjusted $\mathrm{OR}=1.31,95 \% \mathrm{Cl}$, 1.010-1.687 $P_{\text {bonferroni }}=.042$ ).

\footnotetext{
* Correspondence: zhiweiliu@hotmail.com

'International Peace Maternity and Child Health Hospital, School of Medicine, Shanghai Jiao Tong University, $910 \#$ Hengshan Road, Shanghai 20030, China ${ }^{2}$ International Peace Maternity and Child Health Hospital of China Welfare Institution, School of Medicine, Shanghai Jiao Tong University, Shanghai, China

Full list of author information is available at the end of the article
}

C C The Author(s). 2021 Open Access This article is licensed under a Creative Commons Attribution 4.0 International License, which permits use, sharing, adaptation, distribution and reproduction in any medium or format, as long as you give appropriate credit to the original author(s) and the source, provide a link to the Creative Commons licence, and indicate if changes were made. The images or other third party material in this article are included in the article's Creative Commons licence, unless indicated otherwise in a credit line to the material. If material is not included in the article's Creative Commons licence and your intended use is not permitted by statutory regulation or exceeds the permitted use, you will need to obtain permission directly from the copyright holder. To view a copy of this licence, visit http://creativecommons.org/licenses/by/4.0/ The Creative Commons Public Domain Dedication waiver (http://creativecommons.org/publicdomain/zero/1.0/) applies to the data made available in this article, unless otherwise stated in a credit line to the data. 
Conclusions: Maternal vitamin D deficiency $(25(\mathrm{OH}) \mathrm{D}<50 \mathrm{nmol} / \mathrm{L})$ was prevalent in eastern coastal China. The incidence rate of GDM as well as preeclampsia was higher in vitamin D insufficient group while vitamin D deficiency group was liable to intrauterine infection when compared with the other two groups. Most importantly, low vitamin D status in the first trimester of pregnancy was a dependent risk factor for admission to NICU. More well-designed perspective researches are necessary to clarify the role of vitamin $D$ in the early stage of pregnancy.

Keywords: Vitamin D, Maternal, Neonatal, Outcomes, NICU

\section{Introduction}

Vitamin D, with a vital role in calcium absorption and bone metabolism, also functioned in cell proliferation and differentiation, affecting the immune system as well [1]. However, it's worrisome that the incidence of vitamin D deficiency (VDD) is prevalent globally, especially in pregnant women [2]. Low vitamin D status (defined as serum 25(OH)D concentrations $<50 \mathrm{nmol} / \mathrm{L}$ ) was found in $33 \%$ of pregnant women in the US and $24 \%$ in Canada, respectively. In Europe, the prevalence of low vitamin D status was from 20 to $77 \%$ [3]. According to the view of Developmental Origin of Health and Disease (DOHaD) theory, the disturbance in the uterine where the fetus is particularly sensitive to chemicals and other stressors, is related to adverse health effects in adult. Therefore, the lack of vitamin D may have effects on maternal and neonatal outcomes. Observational studies indicated an association between low vitamin $\mathrm{D}$ levels and an increased risk of disorders of placental implantation, impaired glucose tolerance, pre-eclampsia, fetal growth retardation, preterm birth, and caesarean section [4,5], while Randomized Controlled Trial (RCT) found contradicted results [6-8]. Despite some shortterm consequences in the newborns, maternal vitamin $\mathrm{D}$ deficiency also have long-term effects as rickets, increased susceptibility to respiratory illness, autoimmune diseases and type 1 diabetes [9, 10]. Considered the important but controversial role of vitamin $\mathrm{D}$, we aimed to investigate the relationship between maternal vitamin $D$ status in the first trimester of pregnancy and the outcomes of mothers and newborns in order to provide a practical recommendation for clinicians.

\section{Method}

\section{Study design and participants}

In this study, from 2015 to 2016, women followed regular antenatal examinations and gave birth at the International Peace Maternity and Child Health Hospital were included. After excluding cases with incomplete vitamin D and clinical information, diagnosis of multiple pregnancy, 23,394 mothers with their newborns were included ultimately (Fig. 1). Clinical data were extracted from the database and censored by two professional assistants respectively. When disagreement emerged, suggestions from an experienced clinician were considered. All diagnoses were confirmed according to clinical guidelines.

\section{Data collection}

Demographic records were collected at the enrollment along with questionnaires. It consisted of maternal age,

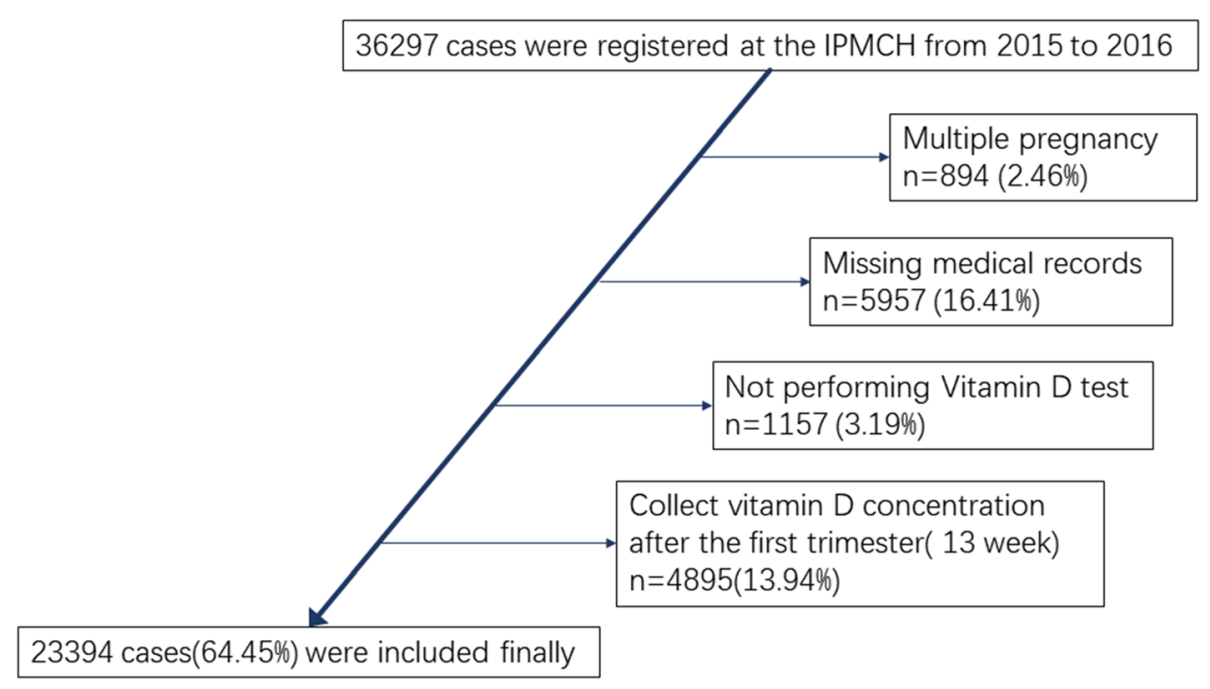

Fig. 1 Flow chart of inclusion criterion of population 
body mass index (BMI) before pregnancy, gravidity or parity. The calculation of gestational age was based on the date of the last menstrual period (MLP), then verified or adjusted by ultrasound reports. Seasons when samples were collected were classified as winter, spring, summer and fall.

\section{Vitamin D level assessment and classification}

We collected maternal fasting blood samples at the first antenatal visit (9-13 weeks of gestation), then transferred it to hospital laboratory, certified by China National Accreditation Service for Conformity Assessment. The blood sample was centrifuged to obtain the serum and stored at $4{ }^{\circ} \mathrm{C}$. Quantitative analysis of vitamin D was performed using chemiluminescence microparticle immunoassay in an Architect I2000SR automatic analyzer (Abbott Diagnostics) with a standard curve following standard clinical procedures by two qualified inspectors. The detection range of vitamin D was 2.00 to $400.00 \mathrm{nmol} / \mathrm{L}$ with both intra- and inter-assay coefficients of variation less than 5\%. According to the Endocrine Society Clinical Practice Guideline [11], mothers with serum 25(OH)D concentration less than 50.00 $\mathrm{nmol} / \mathrm{L}$ were classified as vitamin D deficiency, 50.00 to $75.00 \mathrm{nmol} / \mathrm{L}$ as insufficiency, and more than 75.00 $\mathrm{nmol} / \mathrm{L}$ as sufficiency respectively.

\section{Maternal and neonatal outcomes}

(1) Maternal outcomes:preterm delivery (a live birth before 37 weeks of gestation), pre-eclampsia (high blood pressure and excess protein in the urine after 20 weeks of pregnancy), gestational diabetes (any of the following criteria are meet at a $75 \mathrm{~g}$ oral glucose tolerance test during 24-28 weeks of gestation: fasting: $\quad 92 \mathrm{mg} / \mathrm{dl}, 1 \mathrm{~h}: \quad 180 \mathrm{mg} / \mathrm{dl}, 2 \mathrm{~h}: \quad 153 \mathrm{mg} / \mathrm{dl}$ at a), Intrahepatic cholestasis (pruritus, elevated serum total bile acid (>10 $\mu \mathrm{mol} / \mathrm{L})$ and/or alanine aminotransferase), intrauterine inflammation (pathologic diagnosis of placenta or clinical diagnosis (maternal fever, leukocytosis, maternal and/or fetal tachycardia, uterine tenderness, and preterm rupture of membranes (PROM)).

(2) Neonatal outcomes: birth weight and height, low birth weight $(<2500 \mathrm{~g})$, macrosomia $(>4000 \mathrm{~g})$, small-for-gestational-age (weight $<10$ th percentile or 2SD at birth), score of Apgar 5', NICU hospitalization (for any reasons), asphyxia of newborn, asphyxia of newborn, hyperbilirubinemia, necrotizing enterocolitis, sepsis, death (for any reason).

(3) Criterion for admission to NICU: All the preterm infants, the infants with severe complications, such as visual or hearing impairment, chronic lung disease, temperature instability, hypoglycemia, respiratory distress, hyperbilirubinemia or jaundice, feeding difficulties, urinary tract infection, diarrhea, meningitis and neonatal mortality. All the conditions were diagnosed according to clinical protocols and decisions were made by two experienced neonatologists when infants needed to be transferred to NICU.

\section{Statistical analysis}

All the statistical analyses were performed by the software package SPSS (V25, IBM Corp, Armonk, NY, USA). Continuous data were presented as mean \pm standard deviation (SD) while categorical ones as number (\%). The normality of maternal vitamin $\mathrm{D}$ levels was determined by Kolmogorov-Smirnov test. Distribution of maternal demographic characteristics were compared among groups using the Kruskal-Wallis $H$-test, Pearson's chi-square test or Fisher's exact test based on data characteristic. Bonferroni corrections were applied for multiple comparison correction. Multivariate logistic regression analysis was applied to investigate the relationship between maternal vitamin $\mathrm{D}$ level and maternal and neonatal outcomes. The model was adjusted by maternal age, BMI before pregnancy, gestational weeks and season of blood collection (spring, summer, autumn, winter), delivery mode, number of pre-pregnancies. All $P$ values were 2 -tailed and $P<0.05$ was defined as statistically significant. Odds ratios (ORs) and 95\% confidence intervals (CIs) were applied for the unadjusted and adjusted models.

\section{Results}

\section{General description of vitamin D status}

Totally, 36,297 patients were collected. After the exclusion of 894 (2.46\%) women for multiple pregnancy, 5957 (16.40\%) for missing medical records, 1157 (3.19\%) for not performing Vitamin D test and 4895 (13.49\%) not in the first trimester, results from 22,394 women were finally included in analyses (Fig. 1). The maternal serum $25(\mathrm{OH}) \mathrm{D}$ concentrations in the first trimester was $43.20 \pm 0.10 \mathrm{nmol} / \mathrm{L}$ (mean $\pm \mathrm{SD}$ ) with an overall range of $2.00-124.00 \mathrm{nmol} / \mathrm{L}$ (Table 1, Fig. 2). Of the entire population, 15,696 women $(67.09 \%)$ were $25(\mathrm{OH}) \mathrm{D}$ deficient, 6981(29.84\%) were insufficient and only 2583 (22.2\%) had sufficient 25(OH) D levels (Fig. 3).

\section{Clinical characteristics}

The maternal 25(OH)D levels varied with age, prepregnancy BMI, season when blood was collection, number of previous pregnancy while no interaction was found in the mode of birth, and family history of diabetes or thyroid disease. Women with older age, higher pre-pregnancy $\operatorname{BMI}(P<0.001)$ and less previous pregnancy times $(P=.007)$ indicate a worse $25(\mathrm{OH}) \mathrm{D}$ status. 
Table 1 Distribution of maternal Vitamin D status in the first trimester of pregnancy

\begin{tabular}{|c|c|c|c|c|c|c|c|c|c|c|c|}
\hline Maternal 25(0 & status & & & & & & & & & & \\
\hline Mean(95\%Cl) & Middle & SE & Min & Max & 5th & 10th & 25th & 50th & 75th & 90th & 95th \\
\hline $43.17 \pm .10$ & 42.00 & 15.81 & 2.00 & 124.00 & 20.20 & 23.60 & 30.80 & 42.00 & 53.70 & 64.30 & 70.70 \\
\hline
\end{tabular}

In consistent with seasonal exposure of ultraviolet rays, concentration of vitamin $\mathrm{D}$ fluctuated along with recorded season, with the lowest in winter $(39.40 \pm 15.60$ $\mathrm{nmol} / \mathrm{L})$ and the highest in summer $(47.10 \pm 15.20 \mathrm{nmol} /$ $\mathrm{L}$ ), all were lower than $50 \mathrm{nmol} / \mathrm{L}$ (Table 2).

\section{Maternal outcomes}

Table 3 summarized the maternal outcomes of the population. Interestingly, Women diagnosed as vitamin $\mathrm{D}$ insufficiency had a higher incidence rate of gestational diabetes compared with vitamin D deficiency $(11.90 \%$ vs $10.70 \%, P_{\text {bonferroni }}=.020$ ). The incidence rate of intrauterine infection, preeclampsia were different among groups but not significant after multiple comparison correction. No associations were found between gestational age (both category and numeric values), cesarean section rate, premature rupture of membranes, intrahepatic cholestasis and 2-h postpartum hemorrhage.

\section{Neonatal outcomes}

Most importantly, newborns delivered by women with deficient vitamin D status had a higher incidence rate of admission to NICU (Deficiency: $12.20 \%$ vs Insufficiency: $10.90 \%$ vs Sufficiency: $\left.11.70 \%, P_{\text {bonferroni }}=.002\right)$ and a longer stay (Deficiency: $6.20 \pm 4.10$ vs Insufficiency: $5.90 \pm 3.10$ vs Sufficiency: $5.10 \pm 2.10, P_{\text {bonferroni }}=.010$ ) . Meanwhile, no correlation was observed between maternal vitamin $\mathrm{D}$ status and the birth weight, birth height and other outcomes. (Table 4).

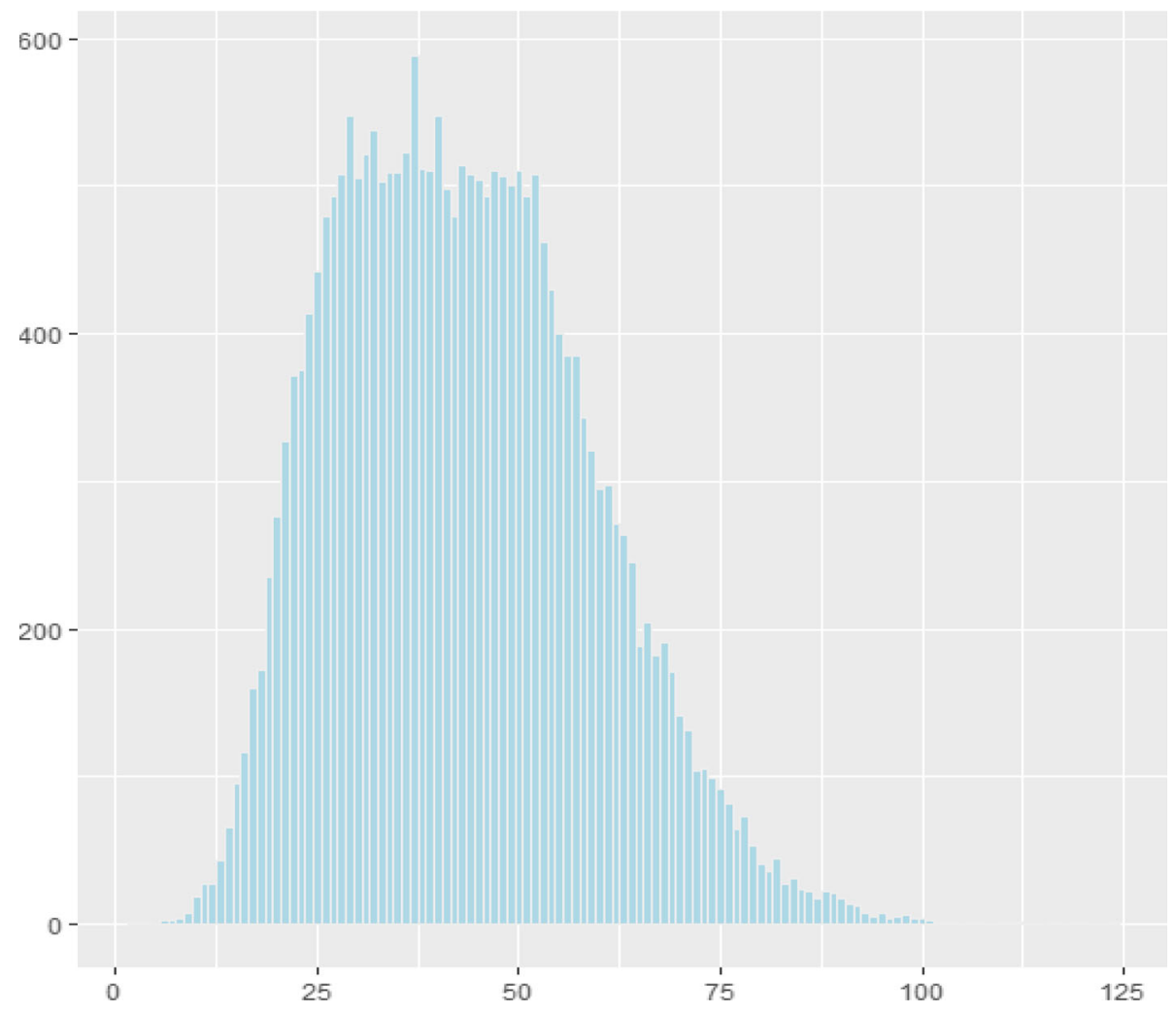

$\mathrm{Y}$ axis: event counts; $\mathrm{X}$ axis: the concentration of maternal serum vitamin $\mathrm{D}$ (nmol/L).

Fig. 2 Distribution of maternal Vitamin D status in the first trimester of pregnancy. $Y$ axis: event counts; $X$ axis: the concentration of maternal serum vitamin $D(\mathrm{nmol} / \mathrm{L})$ 


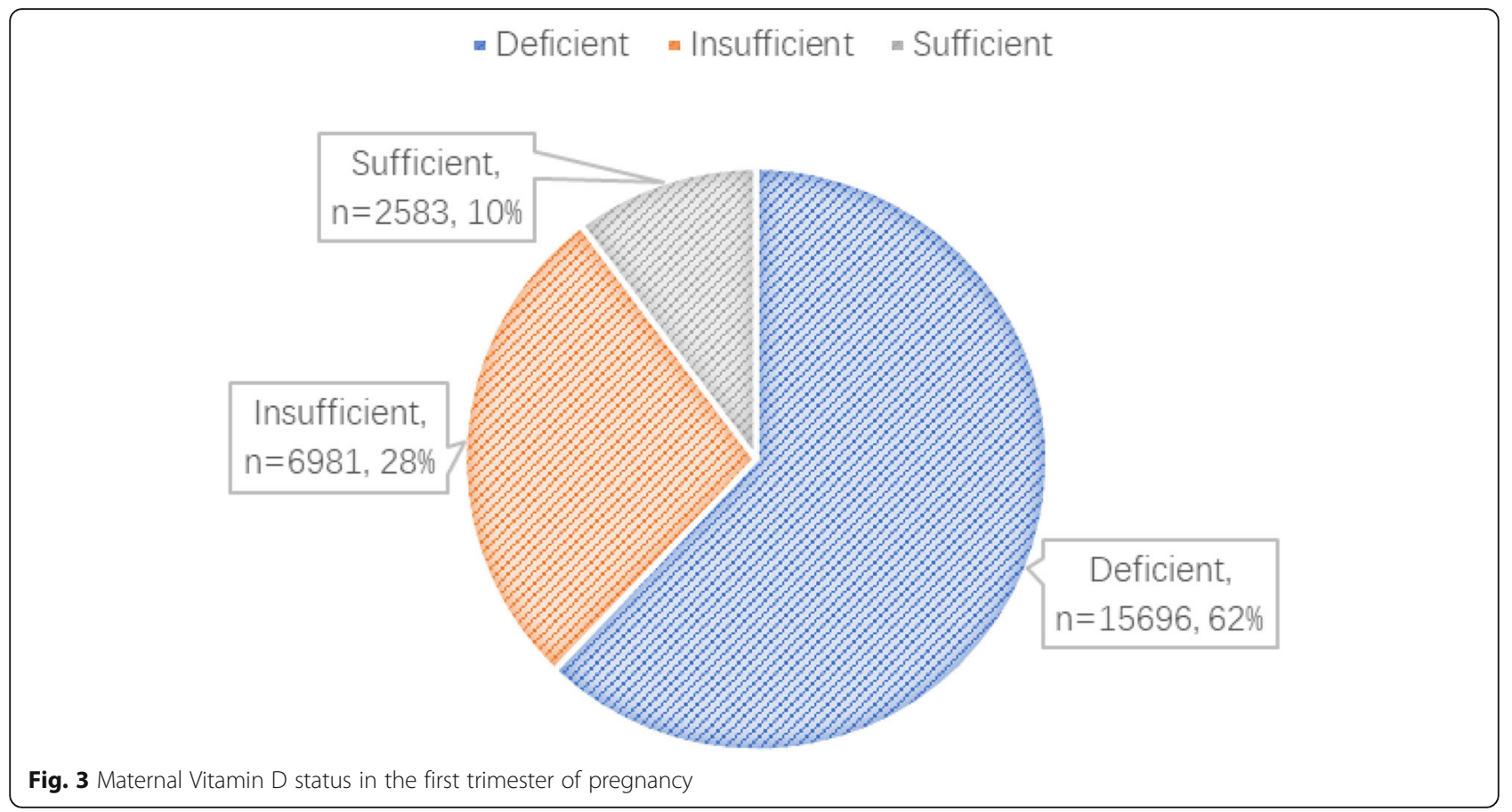

\section{Unadjusted and adjusted risk factors analysis}

Then we burrowed deep into some common complications of mothers and newborns which consist of preterm birth, gestational diabetes, preeclampsia, intrauterine inflammation, cesarean section, premature rupture of membrane, intrahepatic cholestasis for mothers and low birth weight, small for gestational age, large for gestational age, admission to NICU hospitalization, hyperbilirubinemia, necrotizing enterocolitis, sepsis for newborns (Table 5, Fig. 4).

Interestingly, maternal vitamin D deficiency was a dependent risk factor for admission to NICU (unadjusted $\mathrm{OR}=1.350,95 \% \mathrm{CI} \quad(1.045-1.744), \quad P=.022$; adjusted $\mathrm{OR}=1.305,95 \% \mathrm{CI} \quad(1.010-1.687), P=.042)$. To determine the potential confounding factor, we further analyzed demographic baseline of mothers and neonatal outcomes between newborns whether to be admitted to NICU (Table 6). The results indicated that women whose infants were transferred to NICU after delivery had a slightly lower vitamin D concentration $(42.32 \pm 15.18 \mathrm{nmol} / \mathrm{L}$ vs $43.28 \pm 15.89, \quad P=$ $.010)$. Furthermore, lower maternal age $(30.20 \pm 3.50$ vs $30.50 \pm 3.70, P=.006$ ), higher pre-pregnancy BMI $(21.10 \pm 3.40$ vs $20.80 \pm 3.60, P \leq .001)$ and gestational age at birth $(39.00 \pm 1.20$ vs $38.20 \pm 2.40, P=.001)$ was observed in NICU group. NICU group had a lower cesarean section rate $(38.90 \%$ vs $43.50 \%, P \leq .001)$, Apgar score $(9.70 \pm 0.90$ vs $9.90 \pm .59, P<.001)$, birth weight $(3172.00 \pm 619.00$ vs $3366.00 \pm 409.00 P \leq .001)$, and birth length $(49.20 \pm 2.40$ vs $49.90 \pm 1.10$, $P \leq .001)$.
The results also showed that women diagnosed as vitamin $\mathrm{D}$ deficiency had a higher risk for preeclampsia $(\mathrm{OR}=1.851,95 \% \mathrm{CI} \quad(1.013-3.383), P=.045)$. However, the trend was not observed after adjusting for maternal age (category variable), pre-pregnancy BMI (category variable), fetus sex, season of blood sample collection and No. of previous pregnancies. Besides, newborn delivered by vitamin $\mathrm{D}$ deficient women were apt to develop hyperbilirubinemia (unadjusted OR $=1.350$, CI $(1.045-$ $1.744), P=.022$; adjusted $\mathrm{OR}=1.397$, $95 \% \mathrm{CI} \quad(.963-$ 2.027), $P=.079$ ) as well as insufficient group (unadjusted $\mathrm{OR}=1.518, \quad 95 \% \mathrm{CI} \quad(1.042-2.189), \quad P=.031 ; \quad$ adjusted $\mathrm{OR}=1.451,95 \% \mathrm{CI}(.993-2.118), P=.054)$.

\section{Discussion}

In the study, we investigated the maternal vitamin D status in the first trimester of pregnancy and the relations between vitamin $\mathrm{D}$ concentration and maternal as well as neonatal outcomes.

The prevalence of vitamin $D$ deficiency and its risk factors In spite of its importance, the vitamin D status is not optimized among population especially pregnant women who in great amount need of it. Vitamin D deficiency (VDD), defined as serum 25(OH)D concentration < $50.00 \mathrm{nmol} / \mathrm{L}$ ) [11], is prevalent from equatorial areas to Northern Europe, ranging from 26 to 95\% [12-15]. The same trend was observed in our study since 67.09\% women were diagnosed as VDD. Compared with other districts in China, with $90.2 \%$ in Beijing $\left(39.9^{\circ} \mathrm{N}\right)$ [16], $83.6 \%$ in Guiyang $\left(27.2^{\circ} \mathrm{N}\right)$ [17], $18.9 \%$ in Guangzhou 
Table 2 Distribution of maternal demographic characteristics $(N=23,394)$

\begin{tabular}{|c|c|c|c|c|c|}
\hline \multirow{2}{*}{$\begin{array}{l}\text { Maternal } \\
\text { Characteristic }\end{array}$} & \multicolumn{4}{|c|}{ 25(OH)D status (nmol/L) } & \multirow{2}{*}{$\begin{array}{l}\mathrm{X}^{2} / \mathrm{H} \\
P \text { - value }\end{array}$} \\
\hline & $\begin{array}{l}<50 \\
(N=15,696)\end{array}$ & $\begin{array}{l}50-75 \\
(N=6981)\end{array}$ & $\begin{array}{l}>75 \\
(N=717)\end{array}$ & $\begin{array}{l}\text { Total } \\
(\mathrm{N}=23,394)\end{array}$ & \\
\hline Maternal Age(years) & & & & & $\begin{array}{l}59.154 \\
<.001^{*}\end{array}$ \\
\hline$<24^{b}$ & $456(2.90)$ & $178(2.50)$ & $8(1.10)$ & $642(2.70)$ & \\
\hline $25-29^{a}$ & 6894(43.90) & $2807(40.20)$ & 286(39.90) & $9987(42.70)$ & \\
\hline $30-34^{\mathrm{ab}}$ & $6319(40.30)$ & $2928(41.90)$ & 294(41.00) & $9541(40.80)$ & \\
\hline$\geq 35^{\mathrm{ac}}$ & $2027(12.90)$ & $1068(15.30)$ & 129(18.00) & $3224(13.80)$ & \\
\hline Pre-pregnancy BMI $\left(\mathrm{kg} / \mathrm{m}^{2}\right)$ & & & & & $\begin{array}{l}141.771 \\
<.001^{*}\end{array}$ \\
\hline$<18.5^{\mathrm{abc}}$ & 1932(12.30) & $1037(14.90)$ & $170(23.70)$ & $3139(13.40)$ & \\
\hline $18.5-23.9^{b c}$ & $11,448(72.90)$ & $5142(73.70)$ & $493(68.80)$ & $17,083(73.00)$ & \\
\hline$\geq 24^{\mathrm{abc}}$ & $2316(14.80)$ & $802(11.50)$ & $54(7.50)$ & $3172(13.60)$ & \\
\hline Season of blood collection & & & & & $\begin{array}{l}397.256 \\
<.001^{*}\end{array}$ \\
\hline Spring & $4087(26.00)$ & $1620(23.20)$ & $171(23.8)$ & $5878(25.10)$ & \\
\hline Summer & $3268(20.80)$ & $2055(29.40)$ & 229(31.9) & $5552(23.70)$ & \\
\hline Autumn & $3835(24.40)$ & $1928(27.60)$ & $221(29.4)$ & $5974(25.50)$ & \\
\hline Winter & $4506(28.70)$ & 1378(19.70) & 106(14.8) & $5990(25.60)$ & \\
\hline No. of previous pregnancies & & & & & $\begin{array}{l}14.132 \\
.007^{*}\end{array}$ \\
\hline 0 & $10,251(63.50)$ & $4433(63.50)$ & $451(62.90)$ & $15,135(64.70)$ & \\
\hline 1 & $3817(24.30)$ & $1732(24.80)$ & 173(24.10) & $5722(24.50)$ & \\
\hline 2 & $1628(10.40)$ & $816(11.70)$ & $93(13.00)$ & $2537(10.80)$ & \\
\hline Male fetus & $8033(51.20)$ & $3649(52.30)$ & $381(53.10)$ & $12,306(51.60)$ & $\begin{array}{l}3.040 \\
.219^{*}\end{array}$ \\
\hline Family history of diabetes & 1314(8.40) & $579(8.30)$ & $56(7.80)$ & 1914(8.33) & $\begin{array}{l}.301 \\
.860^{*}\end{array}$ \\
\hline Family history of thyroid disease & 3 & 7 & 0 & - & - \\
\hline Maternal Age(years) (Mean \pm SD) ${ }^{\text {ab }}$ & $30.30 \pm 3.64$ & $30.64 \pm 3.70$ & $30.93 \pm 3.69$ & $30.42 \pm 3.66$ & $\begin{array}{l}58.510 \\
<.001^{\#}\end{array}$ \\
\hline Pre-pregnancy BMI $\left(\mathrm{kg} / \mathrm{m}^{2}\right)(\text { Mean } \pm \mathrm{SD})^{\mathbf{a b c}}$ & $21.23 \pm 2.77$ & $20.87 \pm 2.62$ & $20.15 \pm 2.25$ & $21.09 \pm 2.72$ & $\begin{array}{l}58.643 \\
<.001^{\#}\end{array}$ \\
\hline
\end{tabular}

* chi-square test; \# Kruskal-Wallis $\mathrm{H}$-test; Multiple comparison were adjusted with Bonferroni calculation

a. Indicates significant differences between deficient group ( $<50 \mathrm{nmol} / \mathrm{L})$ and insufficient group $(50-75 \mathrm{nmol} / \mathrm{L})$;

b. Indicates significant differences between deficient group and sufficient group $(>75 \mathrm{nmol} / \mathrm{L})$;

c. Indicates significant differences between insufficient group and sufficient group;

$\left(23^{\circ} \mathrm{N}\right)$ [18]. Geographic position, dietary structure, character of job might contribute to the disparity. Why does VDD happen so frequently? Previous researches have unveiled that vitamin $\mathrm{D}$ can be obtained from diverse plant and animal dietary sources as well as sunlight exposure. Then, all sources of vitamin $\mathrm{D}$ are transformed into 25 hydroxyvitamin $\mathrm{D}$, the predominant but inactive circulating form of vitamin $\mathrm{D}$, in the liver by 25 hydroxylases. CYP27B1(1-alpha-hydroxylase) in the kidney mainly and other sites including placenta and brain convert $25(\mathrm{OH}) \mathrm{D}$ into $1,25(\mathrm{OH})_{2} \mathrm{D}[19,20]$ which induce both genomic and non-genomic effects mediated by VDR [21, 22]. Considered the comprehensive metabolic pathways, dietary depletion, seasonal sunlight exposure lacking, adiposity and genetic variants contribute to the incidence of VDD [23]. For instance, the negative correlation between vitamin D status and BMI in the study could be explained by a relatively smaller skin surface for vitamin D synthesis [24]. In addition, since vitamin $\mathrm{D}$ is a fat-soluble molecular, it might be stored in fat tissue instead of being detecting as free form. According to Chen, each additional unit $\left(1 \mathrm{~kg} / \mathrm{m}^{2}\right)$ of prepregnancy BMI indicated a $0.23 \mathrm{ng} / \mathrm{mL}$ increase in $25(\mathrm{OH}) \mathrm{D}$ concentration [25]. Interestingly, in our study, 
Table 3 Maternal outcomes stratified by maternal vitamin D status in the first trimester

\begin{tabular}{|c|c|c|c|c|c|}
\hline \multirow{2}{*}{$\begin{array}{l}\text { Maternal } \\
\text { Outcomes }\end{array}$} & \multicolumn{4}{|c|}{ 25(OH)D status(nmol/L) } & \multirow{2}{*}{$\begin{array}{l}\mathrm{X}^{2} / \mathrm{F} / \mathrm{H} \\
P \text { - value }\end{array}$} \\
\hline & $\begin{array}{l}<50 \\
(N=15,696)\end{array}$ & $\begin{array}{l}50-74.9 \\
(\mathrm{~N}=6981)\end{array}$ & $\begin{array}{l}\geq 75 \\
(N=717)\end{array}$ & $\begin{array}{l}\text { Total } \\
(\mathrm{N}=23,394)\end{array}$ & \\
\hline Gestational Age (weeks) (Mean \pm SD) ${ }^{a}$ & $38.9 \pm 1.4$ & $38.9 \pm 1.4$ & $38.8 \pm 1.2$ & $38.9 \pm 1.4$ & $\begin{array}{l}.327 \\
.036^{\&}\end{array}$ \\
\hline Gestational Age(weeks) (Category) & & & & & $\begin{array}{l}8.023 \\
.431^{*}\end{array}$ \\
\hline$<28$ & 3 & 0 & 0 & 3 & \\
\hline $28-31+6$ & $54(0.3)$ & 26(0.4) & 0 & $80(0.3)$ & \\
\hline $32-33+6$ & $101(0.6)$ & $31(0.4)$ & $4(0.6)$ & 136(0.6) & \\
\hline $34-36+6$ & $692(4.4)$ & $324(4.6)$ & $32(4.5)$ & 1048(4.5) & \\
\hline$\geq 37$ & $14,846(94.6)$ & $6600(94.5)$ & $681(95.0)$ & $22,127(94.6)$ & \\
\hline Cesarean section & $6715(42.8)$ & $3028(43.4)$ & $301(42.0)$ & $10,044(42.9)$ & $\begin{array}{l}0.969 \\
.616^{*}\end{array}$ \\
\hline Premature rupture of membranes & $18(0.1)$ & $12(0.2)$ & 0 & $30(0.1)$ & $\begin{array}{l}2.185 \\
.335^{*}\end{array}$ \\
\hline Gestational diabetes $^{a}$ & $1680(10.7)$ & $831(11.9)$ & 73(10.2) & 2584(11.0) & $\begin{array}{l}7.648 \\
.022^{*}\end{array}$ \\
\hline Preeclampsia & $440(1.9)$ & $162(2.3)$ & $11(1.5)$ & $613(2.6)$ & $\begin{array}{l}7.832 \\
.020^{*}\end{array}$ \\
\hline Intrahepatic cholestasis & 108(0.7) & $38(0.5)$ & $8(1.1)$ & $154(0.7)$ & $\begin{array}{l}3.894 \\
.143^{*}\end{array}$ \\
\hline Intrauterine infection & $330(2.1)$ & 115(1.6) & $10(1.4)$ & 455(1.9) & $\begin{array}{l}6.422 \\
.040^{*}\end{array}$ \\
\hline 2-h postpartum hemorrhage $(\mathrm{ml})$ & $256 \pm 149$ & $255 \pm 106$ & $244 \pm 84$ & $255 \pm 137$ & $\begin{array}{l}.001 \\
.797^{\#}\end{array}$ \\
\hline
\end{tabular}

* chi-square test; \# Kruskal-Wallis $\mathrm{H}$-test; \& ANOVA. Multiple comparison was adjusted with Bonferroni calculation

a. Indicates significant differences between deficient group $(<50 \mathrm{nmol} / \mathrm{L})$ and insufficient group $(50-75 \mathrm{nmol} / \mathrm{L})$;

b. Indicates significant differences between deficient group and sufficient group $(>75 \mathrm{nmol} / \mathrm{L})$;

c. Indicates significant differences between insufficient group and sufficient group;

the level of Vitamin D increased with maternal age and the number of previous pregnancies. It may due to that multipara had experience in nutrient supplements, lifestyle changes and healthcare services requirements than novice mothers.

\section{Maternal and neonatal outcomes}

Not only does maternal VDD associates with increased risk of gestational diabetes and pre-eclampsia [26, 27], but also directly effects on offspring health as low birth weight, impaired brain development, obesity and insulin resistance [28-30].

\section{Admission to NICU}

Of the most important, we found the incidence of newborns admitted to NICU was strongly associated with maternal vitamin D status in the first trimester of pregnancy. When compared with women with sufficient serum vitamin $\mathrm{D}$ concentration $(\geq 75 \mathrm{nmol} / \mathrm{L})$, women diagnosed as insufficiency $(50-74.9 \mathrm{nmol} / \mathrm{L})$ and deficiency $(<50 \mathrm{nmol} / \mathrm{L})$ had higher risk of delivering babies admitted to NICU in both unadjusted and adjusted models (Deficiency: unadjusted $\mathrm{OR}=1.350, \quad 95 \% \mathrm{CI}$ (1.045-1.744), $P=.022$, adjusted $\mathrm{OR}=1.305,95 \% \mathrm{CI}$ (1.010-1.687), $P<0.001)$. Meanwhile, the result revealed a trend that the risk of NICU hospitalization of newborns increased as the maternal vitamin D status deteriorated. Newborns admitted to NICU were premature or companied with severe complications such as septicemia, hypoxic-ischemic encephalopathy (HIE), necrotizing enterocolitis [31]. In the one hand, some disease may have long-term sequela (malformation, Neurodevelopmental abnormality). However, newborns might be transferred to NICU for diverse reasons, it can be a practical indicator for both prognostic and economic consequences. Neonatal intensive care (NIC) cost 26.2 billion USD a year in the United States [32]. With escalating health expenditure, resource allocation by the government or public sector will be determined by health economic evaluations of new technologies or innovations. Since vitamin D supplementation was convenient and effective to reduce the incidence of NICU hospitalization, our research provided a practical recommendation for decision-makers. What needs to be 
Table 4 Neonatal outcomes stratified by maternal vitamin D status in the first trimester

\begin{tabular}{|c|c|c|c|c|c|}
\hline \multirow{2}{*}{$\begin{array}{l}\text { Neonatal } \\
\text { Outcomes }\end{array}$} & \multicolumn{4}{|c|}{ Maternal 25(OH)D status(nmol/L) } & \multirow{2}{*}{$\begin{array}{l}\mathrm{X}^{2} / \mathrm{F} / \mathrm{H} \\
P \text {-value }\end{array}$} \\
\hline & $\begin{array}{l}<50 \\
(N=15,696)\end{array}$ & $\begin{array}{l}50-75 \\
(\mathrm{~N}=6981) \\
\end{array}$ & $\begin{array}{l}>75 \\
(\mathrm{~N}=717)\end{array}$ & $\begin{array}{l}\text { Total } \\
(\mathrm{N}=23,394)\end{array}$ & \\
\hline Birth weight (Mean \pm SD) & $3346 \pm 448$ & $3340 \pm 437$ & $3329 \pm 412$ & $3343 \pm 443$ & $\begin{array}{l}.833 \\
.435^{\&}\end{array}$ \\
\hline Birth weight z score (Mean \pm SD) & $.005 \pm 1.010$ & $-.008 \pm .985$ & $-.032 \pm .928$ & - & \\
\hline Low birth weight: $<2500 \mathrm{~g}$ & $372(2.4)$ & $141(2.0)$ & $14(2.0)$ & $527(2.3)$ & $\begin{array}{l}2.995 \\
.224^{*}\end{array}$ \\
\hline Very low birth weight: $<1500 \mathrm{~g}$ & $42(0.3)$ & 20(0.3) & $1(0.1)$ & $63(0.3)$ & $\begin{array}{l}0.529 \\
.768^{*}\end{array}$ \\
\hline Small for gestational age: $<10$ th percentile ( 3 case excluded) & $322(2.1)$ & 135(1.9) & $15(2.1)$ & $472(2.0)$ & $\begin{array}{l}1.833 \\
.766^{*}\end{array}$ \\
\hline High birth weight: > $4000 \mathrm{~g}$ & $955(6.1)$ & $390(5.6)$ & $38(5.3)$ & 1383(5.9) & $\begin{array}{l}2.650 \\
.266^{*}\end{array}$ \\
\hline Large for gestational age: >90th percentile & $3679(23.4)$ & 1612(23.1) & $160(22.3)$ & $5451(23.3)$ & $\begin{array}{l}2.212 \\
.697^{*}\end{array}$ \\
\hline Birth length (Mean \pm SD) & $49.9 \pm 1.4$ & $49.8 \pm 1.4$ & $49.9 \pm 1.1$ & $49.9 \pm 1.4$ & $\begin{array}{l}.689 \\
.502^{\&}\end{array}$ \\
\hline Apgar score (Mean \pm SD) & $9.7 \pm .9$ & $9.7 \pm 1.0$ & $9.7 \pm .8$ & $9.7 \pm .9$ & $\begin{array}{l}1.938 \\
.144^{\&}\end{array}$ \\
\hline Apgar score & & & & & $\begin{array}{l}7.144 \\
.308^{*}\end{array}$ \\
\hline $0-3$ & $28(.2)$ & $17(.2)$ & 0 & $45(.2)$ & - \\
\hline $4-6$ & $106(.7)$ & $43(.6)$ & 2(.3) & $151(.6)$ & \\
\hline $7-8$ & $270(1.7)$ & $137(2.0)$ & $9(1.3)$ & $416(1.8)$ & \\
\hline $9-10$ & $15,292(97.4)$ & $6784(97.2)$ & 706(98.5) & $22,782(97.4)$ & \\
\hline Admission to neonatal intensive care unit & 1918(12.2) & $761(10.9)$ & $67(9.3)$ & $2746(11.7)$ & $\begin{array}{l}12.200 \\
.002^{*}\end{array}$ \\
\hline Admission to neonatal intensive care unit (Mean \pm SD) & $6.2 \pm 4.1$ & $5.9 \pm 3.1$ & $5.1 \pm 2.1$ & $6.1 \pm 3.2$ & $.010^{\#}$ \\
\hline Any respiratory disorders" & $334(2.1)$ & $131(1.9)$ & $16(2.2)$ & $481(2.1)$ & $\begin{array}{l}1.205 \\
.300^{*}\end{array}$ \\
\hline Dyspnea (clinical diagnosis) & $8(0.1)$ & $5(0.1)$ & 0 & $13(0.1)$ & $\begin{array}{l}.782 \\
.676^{*}\end{array}$ \\
\hline Wet lung & $266(1.7)$ & $107(1.5)$ & 13(1.8) & $386(1.6)$ & $\begin{array}{l}.902 \\
.637^{*}\end{array}$ \\
\hline Aspiration pneumonia & 5 & 3 & 0 & 8 & - \\
\hline Pulmonary arterial hypertension & 2 & 0 & 0 & 2 & - \\
\hline Pneumothorax & $30(0.2)$ & $6(0.1)$ & $1(0.1)$ & $37(0.2)$ & $\begin{array}{l}3.402 \\
.183^{*}\end{array}$ \\
\hline Bronchopulmonary dysplasia & 2 & 0 & 0 & 2 & - \\
\hline Neonatal convulsion & 6 & 6 & 1 & 13 & - \\
\hline Hyperbilirubinemia & $971(6.2)$ & $434(6.2)$ & $30(4.2)$ & 1435(6.1) & $\begin{array}{l}4.487 \\
.087^{*}\end{array}$ \\
\hline Necrotizing enterocolitis & $79(0.5)$ & $26(0.4)$ & $4(0.6)$ & $109(0.5)$ & $\begin{array}{l}1.919 \\
.383^{*}\end{array}$ \\
\hline Sepsis & $29(0.2)$ & $14(0.2)$ & $2(0.3)$ & $45(0.2)$ & - \\
\hline Retinopathy of prematurity & 19 & 7 & 0 & 26 & - \\
\hline Neonatal death & 1 & 1 & 0 & 2 & - \\
\hline
\end{tabular}

* chi-square test; \# Kruskal—Wallis $H$-test; \& ANOVA. Bonferroni adjustment was performed

Multiple comparisons were adjusted with Bonferroni calculation. १ any respiratory disorders (including dyspnea, wet lung, aspiration pneumonia,

pulmonary arterial hypertension, pneumothorax, bronchopulmonary dysplasia);

a. Indicates significant differences between deficient group $(<50 \mathrm{nmol} / \mathrm{L})$ and insufficient group $(50-75 \mathrm{nmol} / \mathrm{L})$;

b. Indicates significant differences between deficient group and sufficient group $(>75 \mathrm{nmol} / \mathrm{L})$;

c. Indicates significant differences between insufficient group and sufficient group; 
Table 5 Unadjusted and adjusted odds ratios for maternal and neonatal stratified by vitamin D status

\begin{tabular}{|c|c|c|c|c|c|c|}
\hline \multirow[b]{2}{*}{ Maternal outcomes } & \multicolumn{3}{|c|}{ Unadjusted OR $(95 \% \mathrm{Cl})$ and $P$ Value } & \multicolumn{3}{|c|}{ Adjusted $\mathrm{OR}^{\mathrm{a}}(95 \% \mathrm{Cl})$ And $P$ Value } \\
\hline & $\geq 75$ & $50-74.9$ & $<50$ & $\geq 75$ & $50-74.9$ & $<50$ \\
\hline Preterm birth & $\begin{array}{l}1.00 \\
-\end{array}$ & $\begin{array}{l}1.092(.769-1.551) \\
.623\end{array}$ & $\begin{array}{l}1.086(.771-1.529) \\
.637\end{array}$ & $\begin{array}{l}1.00 \\
-\end{array}$ & $\begin{array}{l}1.080(.760-1.535) \\
.667\end{array}$ & $\begin{array}{l}1.078(.765-1.520) \\
.667\end{array}$ \\
\hline Gestational diabetes & $\begin{array}{l}1.00 \\
-\end{array}$ & $\begin{array}{l}1.192(.926-1.535) \\
.173\end{array}$ & $\begin{array}{l}1.057(.826-1.354) \\
.658\end{array}$ & $\begin{array}{l}1.00 \\
-\end{array}$ & $\begin{array}{l}1.145(.886-1.478) \\
.300\end{array}$ & $\begin{array}{l}1.014(.789-1.303) \\
.915\end{array}$ \\
\hline Preeclampsia & $\begin{array}{l}1.00 \\
-\end{array}$ & $\begin{array}{l}1.525(.824-2.822) \\
.179\end{array}$ & $\begin{array}{l}1.851(1.013-3.383) \\
.045\end{array}$ & $\begin{array}{l}1.00 \\
-\end{array}$ & $\begin{array}{l}1.387(.748-2.571) \\
.299\end{array}$ & $\begin{array}{l}1.609(.879-2.948) \\
.123\end{array}$ \\
\hline Intrauterine inflammation & $\begin{array}{l}1.00 \\
-\end{array}$ & $\begin{array}{l}1.184(.618-2.270) \\
.611\end{array}$ & $\begin{array}{l}1.518(.806-2.861) \\
.196\end{array}$ & $\begin{array}{l}1.00 \\
-\end{array}$ & $\begin{array}{l}1.187(.618-2.278) \\
.607\end{array}$ & $\begin{array}{l}1.545(.817-2.920) \\
.181\end{array}$ \\
\hline Cesarean section & $\begin{array}{l}1.00 \\
-\end{array}$ & $\begin{array}{l}1.059(.906-1.237) \\
.437\end{array}$ & $\begin{array}{l}1.033(.888-1.203) \\
.672\end{array}$ & $\begin{array}{l}1.00 \\
-\end{array}$ & $\begin{array}{l}1.049(.895-1.230) \\
.556\end{array}$ & $\begin{array}{l}1.040(.890-1.214) \\
.622\end{array}$ \\
\hline Intrahepatic cholestasis & $\begin{array}{l}1.00 \\
-\end{array}$ & $\begin{array}{l}.485(.225-1.044) \\
.064\end{array}$ & $\begin{array}{l}.614(.298-1.264) \\
.186\end{array}$ & $\begin{array}{l}1.00 \\
-\end{array}$ & $\begin{array}{l}.501(.232-1.079) \\
.078\end{array}$ & $\begin{array}{l}.633(.306-1.308) \\
.217\end{array}$ \\
\hline \multicolumn{7}{|l|}{ Neonatal outcomes } \\
\hline Low birth weight & $\begin{array}{l}1.00 \\
-\end{array}$ & $\begin{array}{l}1.035(.594-1.803) \\
.903\end{array}$ & $\begin{array}{l}1.219(.711-2.090) \\
.471\end{array}$ & $\begin{array}{l}1.00 \\
-\end{array}$ & $\begin{array}{l}1.055(.606-1.839) \\
.849\end{array}$ & $\begin{array}{l}1.263(.736-2.169) \\
.397\end{array}$ \\
\hline Small for gestational age & $\begin{array}{l}1.00 \\
-\end{array}$ & $\begin{array}{l}.923(.538-1.582) \\
.770\end{array}$ & $\begin{array}{l}.980(.581-1.654) \\
.941\end{array}$ & $\begin{array}{l}1.00 \\
-\end{array}$ & $\begin{array}{l}.991(.822-1.196) \\
.927\end{array}$ & $\begin{array}{l}.999(.832-1.199) \\
.989\end{array}$ \\
\hline Large for gestational age & $\begin{array}{l}1.00 \\
-\end{array}$ & $\begin{array}{l}1.045(.869-1.257) \\
.638\end{array}$ & $\begin{array}{l}1.066(.891-1.276) \\
.485\end{array}$ & $\begin{array}{l}1.00 \\
-\end{array}$ & $\begin{array}{l}.978(.569-1.678) \\
.935\end{array}$ & $\begin{array}{l}1.060(.626-1.793) \\
.829\end{array}$ \\
\hline Admission to neonatal intensive care unit & $\begin{array}{l}1.00 \\
-\end{array}$ & $\begin{array}{l}1.187(.913-1.543) \\
.201\end{array}$ & $\begin{array}{l}1.350(1.045-1.744) \\
.022\end{array}$ & $\begin{array}{l}1.00 \\
-\end{array}$ & $\begin{array}{l}1.160(.892-1.509) \\
.269\end{array}$ & $\begin{array}{l}1.305(1.010-1.687) \\
.042\end{array}$ \\
\hline Any respiratory disorders & $\begin{array}{l}1.00 \\
-\end{array}$ & $\begin{array}{l}.838(.496-1.416) \\
.509\end{array}$ & $\begin{array}{l}.950(.572-1.578) \\
.842\end{array}$ & $\begin{array}{l}1.00 \\
-\end{array}$ & $\begin{array}{l}.824(.487-1.394) \\
.470\end{array}$ & $\begin{array}{l}.937(.563-1.559) \\
.803\end{array}$ \\
\hline Hyperbilirubinemia & $\begin{array}{l}1.00 \\
-\end{array}$ & $\begin{array}{l}1.518(1.042-2.189) \\
.031\end{array}$ & $\begin{array}{l}1.510(1.042-2.189) \\
.030\end{array}$ & $\begin{array}{l}1.00 \\
-\end{array}$ & $\begin{array}{l}1.451(.993-2.118) \\
.054\end{array}$ & $\begin{array}{l}1.397(.963-2.027) \\
.079\end{array}$ \\
\hline Necrotizing enterocolitis & $\begin{array}{l}1.00 \\
-\end{array}$ & $\begin{array}{l}.666(.232-1.915) \\
.451\end{array}$ & $\begin{array}{l}.902(.329-2.469) \\
.840\end{array}$ & $\begin{array}{l}1.00 \\
-\end{array}$ & $\begin{array}{l}.684(.238-1.969) \\
.482\end{array}$ & $\begin{array}{l}.947(.345-2.603) \\
.916\end{array}$ \\
\hline Sepsis & $\begin{array}{l}1.00 \\
-\end{array}$ & $\begin{array}{l}.718(.163-3.167) \\
.662\end{array}$ & $\begin{array}{l}.662(.158-2.779) \\
.662\end{array}$ & $\begin{array}{l}1.00 \\
-\end{array}$ & $\begin{array}{l}.668(.151-2.951) \\
.594\end{array}$ & $\begin{array}{l}.597(.141-2.523) \\
.483\end{array}$ \\
\hline
\end{tabular}

${ }^{a}$ Adjusted for maternal age (category variable), pre-pregnancy BMI (category variable), fetus sex, collection season of blood sample, No. of previous pregnancies. Using vitamin D sufficiency $(>75 \mathrm{nmol} / \mathrm{L})$ as a reference

confirmed is how to intervene, for vitamin D may plays different roles across the pregnancy, from placenta implantation to fetal bone formation.

\section{Preterm birthPreterm birth}

Globally, $11 \%$ of newborns are preterm birth, leading to 15 million premature infants. The incidence of preterm birth is increasing in many countries, meanwhile, the survival rate of preterm babies has dramatically improved in developed countries [33, 34]. Preterm birth represents a significant cause of death and can lead to serious harm to survivors all around the world [35]. In our study, maternal vitamin $\mathrm{D}$ status had no correlation with preterm birth. Nonetheless, a possible negative correlation between maternal vitamin D status and preterm birth was reported $[36,37]$. A recent meta-analysis consisted of 24 observational studies revealed the association between low vitamin D levels $(<50.00 \mathrm{nmol} / \mathrm{L})$ and increased risk of preterm birth $(\mathrm{OR}=1.58,95 \% \mathrm{CI}$ (1.08 to 2.31$)$ [38-41] while studies from New Zealand [42] and China [43] shared the similar view with us. The discrepancy may due to different sample size, research methods confounding factors, various $25(\mathrm{OH}) \mathrm{D}$ cutoff values, population characteristics and methods for measuring vitamin D status. Since we haven't excluded some cases with risk factors related to PTB, the certain relation might be concealed. In addition, molecular research demonstrated that vitamin D metabolism can be affected by single nucleotide polymorphisms (SNP) of VDR genes such as BsmI, FokI, TaqI, and ApaI, resulting in different maternal serum vitamin $D$ concentrations and functions in downstream, even given the same amount of supplement [44].

\section{Gestational diabetes mellitus}

GDM, manifests as insulin resistance, increased inflammatory factors and oxidative stress [45], can result in adverse maternal outcomes and long-term sequelae in the offspring [46]. A RCT in Iran investigated that vitamin D supplementation in high-risk pregnancies women leaded to a significant reduction in fasting plasma 

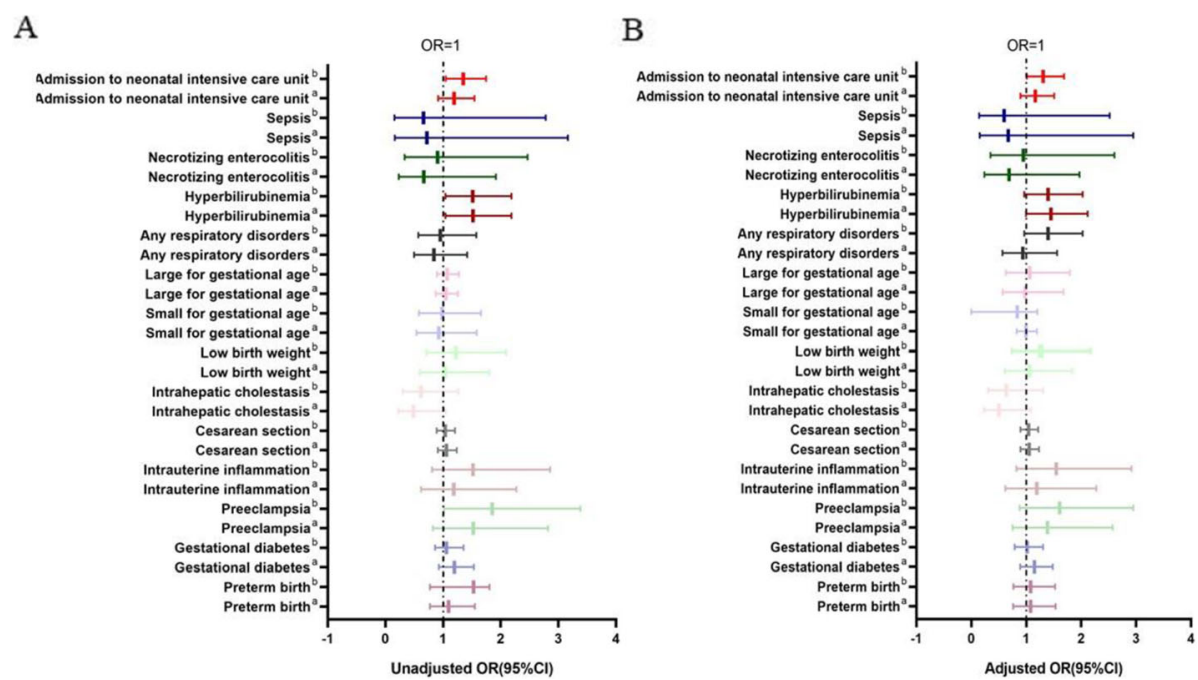

Fig. 4 The Forest Plot of unasjusted and adjusted models. A. The unadjusted model. B. The adjusted model (Adjusted for maternal age (category variable), pre-pregnancy BMI (category variable), fetus sex, collection season of blood sample, No. of previous pregnancies. Using vitamin D sufficiency (> $75 \mathrm{nmol} / \mathrm{L}$ ) as a reference. a. Insufficient group vs sufficient group. b. Deficient group vs sufficient group. The dot line indicates where OR=1

glucose concentration, insulin levels and HOMA-IR accompanied with lower serum LDL-cholesterol and total cholesterol [7]. However, a meta-analysis including several RCTs found no beneficial effect of vitamin D supplementation on indicators of glucose homeostasis such as fasting plasma glucose (FPG), insulin, HbA1cand lipo-metabolism spectrum [47]. In this study, we found the correlation between maternal vitamin D status and GDM was not noted in unadjusted and adjusted models. Researches revealed vitamin D can intervene glucose homeostasis from different layers: immune cell infiltration among glandular cells results in inflammation and functional pancreatic alteration. As an anti-inflammatory molecular, vitamin D can rebuild insulin secretion function to some extent [48]. Through indirect way, vitamin $\mathrm{D}$ increase duodenal absorption and renal resorption of calcium, a vital downstream factor of insulin pathway [49]. Moreover, vitamin D receptors (VDRs) were found participating in promoting insulin sensitivity [50]. For clinician and policymakers, the role of vitamin D supplementation in gestational diabetes does not come into light. [51]. Fortunately, some double-blind RCTs indicated beneficial effect of vitamin $\mathrm{D}$ during the first and second trimesters of pregnancy supplementation on GDM [23].

\section{Preeclampsia}

In the study, Preeclampsia was not an independent risk factor whether adjusted for confounding factors. In consistent with our study, few observational studies showed negative results for the effects of vitamin $D$ on preeclampsia [52]. However, a meta-analysis consists of 12 studies indicated that women with low maternal serum
$25(\mathrm{OH}) \mathrm{D}$ concentrations were susceptible to preeclampsia. Although the poor quality of evidence raised vagueness in causality [53]. The same results were reported in another recently updated meta-analysis including 23 studies with slightly increased fixed (1.33) [54]. The disagreement of these researches may result from different time when the blood samples were collected, since vitamin D has diverse roles throughout the whole pregnancy including the regulation of trophoblast differentiation and EVT invasion of the decidua and myometrium at early stage [55].

\section{Intrauterine inflammation}

Intrauterine inflammation is often related to chorioamnionitis, a common cause of preterm birth leading to adverse neonatal outcomes [56, 57]. If not treated timely and properly, long-term outcomes as neurodevelopmental sequelae and chronic lung disease might influence life quality in adulthood [58, 59]. Chorioamnionitis may be classified as clinical chorioamnionitis and subclinical/histologic chorioamnionitis based on clinical signs and laboratory evidences. A recent observational study suggested that vitamin $\mathrm{D}$ in early pregnancy was a protective factor for intrauterine infection and neonatal sepsis was associated since it reduced placental inflammation [14]. According to our results, no correlation was found between intrauterine inflammation and vitamin $D$. It might be due to the reason that we confirmed the diagnosis based on clinical signs other than histological evidence. Besides, the etiology of chorioamnionitis is 
Table 6 Characteristics of newborns admitted to NICU ( $N=2746)$

\begin{tabular}{ll}
\hline $\begin{array}{ll}\text { Maternal characteristics and } \\
\text { Pregnant outcomes }\end{array}$ & Admission to \\
\cline { 2 - 2 } & Without $(\boldsymbol{N}=$ \\
\hline 25(OH)D levels (Mean \pm SD) & $43.283 \pm 15.893$ \\
25(OH)D levels (N\%) & \\
$<50^{\mathrm{a}}$ & \\
$50-74.9^{\mathrm{a}}$ & $13,778(66.7)$ \\
$\geq 75^{\mathrm{a}}$ & $6220(30.1)$ \\
Maternal Age(years) & $650(3.1)$ \\
Mean & $30.5 \pm 3.7$
\end{tabular}

Maternal Age(years)

$30.5 \pm 3.7$

Maternal Age(years) (N(\%))

$$
\begin{aligned}
& 628(2.8) \\
& 9775(42.4) \\
& 9351(40.7) \\
& 3165(14.1) \\
& 20.8 \pm 3.6
\end{aligned}
$$

\section{(Mean \pm SD)}

Pre-pregnancy BMI (kg/m) (N(\%))

$$
\begin{aligned}
& <18.5^{\mathrm{a}} \\
& 18.5-23.9 \\
& \geq 24^{\mathrm{a}}
\end{aligned}
$$

Season of blood collection

$$
\begin{aligned}
& \text { Spring } \\
& \text { Summer } \\
& \text { Autumn }
\end{aligned}
$$$$
\text { Winter }
$$

No. of previous pregnancies

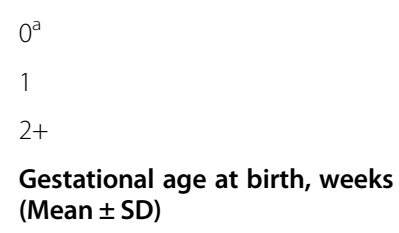

Gestational age at birth, weeks (Mean \pm SD)

Gestational age at birth $(\mathrm{N}(\%))$

$$
\begin{aligned}
& <28 \\
& 28-31+6 \\
& 32-33+6 \\
& 34-36+6 \\
& \geq 37
\end{aligned}
$$

Male (N\%)

Cesarean section (N\%)

2-h postpartum hemorrhage $(\mathrm{ml})$

$$
\begin{aligned}
& 2813(13.6) \\
& 15,105(73.2)
\end{aligned}
$$$$
\text { 2730(13.2) }
$$

$4910(23.8)$

5314 (25.7)

$5292(25.6)$

$$
\begin{aligned}
& 13,311(64.5) \\
& 5072(24.6) \\
& 2265(11.0) \\
& 39.0 \pm 1.2
\end{aligned}
$$

2

$$
32(.2)
$$$$
13(.1)
$$$$
614(3.0)
$$$$
\text { 19,987 (96.8) }
$$$$
10,600 \text { (51.3) }
$$

8975 (43.5)

$$
241 \pm 155
$$

$\mathrm{X}^{2} / \mathrm{Z} /$ $P$-value

$-2.590$

$$
.010^{\&}
$$

12.200

$.002^{*}$

1918 (69.8)

761 (27.7)

$67(2.4)$

$30.2 \pm 3.5$

$-2.759$

$.006^{\&}$

17.364

$.001^{*}$

14 (2.1)

212 (44.9)

$244(41.2)$

$114(11.7)$

$21.1 \pm 3.4$

20.731

$<.001^{\#}$

$326(11.9)$

1978 (72.0)

$442(16.1)$

8.109

$.044^{*}$

$746(27.2)$
$642(23.4)$
$660(24.0)$
$698(25.4)$

1824 (66.4)

650 (23.7)

272 (9.9)

$38.2 \pm 2.4$

$-10.445$

$<.001^{\&}$

1976.277

$<.001^{*}$

1

$48(1.7)$

123 (4.5)

434 (15.8)

2140 (83.5)

1463 (53.3)

3.655
$.056^{*}$

1069 (38.9)

22.366 $<.001^{*}$

$-7.964$ $<.001^{\&}$ 
Table 6 Characteristics of newborns admitted to NICU ( $N=2746)$ (Continued)

\begin{tabular}{|c|c|c|c|}
\hline \multirow{2}{*}{$\begin{array}{l}\text { Maternal characteristics and } \\
\text { Pregnant outcomes }\end{array}$} & \multicolumn{2}{|c|}{ Admission to neonatal intensive care unit } & \multirow{2}{*}{$\begin{array}{l}\mathrm{X}^{2} / \mathrm{Z} / \\
P \text { - value }\end{array}$} \\
\hline & Without $(N=20,648)$ & With $(N=2746)$ & \\
\hline Apgar score(Mean \pm SD) & $9.9 \pm .6$ & $9.7 \pm .9$ & $\begin{array}{l}-18.128 \\
<.001^{\&}\end{array}$ \\
\hline $\begin{array}{l}\text { Apgar score } \\
\text { (N\%) }\end{array}$ & & & $\begin{array}{l}233.297 \\
<.001^{*}\end{array}$ \\
\hline $0-3$ & $37(.2)$ & $8(.3)$ & \\
\hline $4-6^{a}$ & $97(.5)$ & $54(2.0)$ & \\
\hline $7-8^{\mathrm{a}}$ & 289(1.4) & $127(4.6)$ & \\
\hline $9-10^{a}$ & $20,225(98.0)$ & $2557(93.1)$ & \\
\hline Neonatal birth weight(g) & $3366 \pm 409$ & $3172 \pm 619$ & $\begin{array}{l}-12.208 \\
<.001^{\&}\end{array}$ \\
\hline Neonatal birth height(cm) & $49.9 \pm 1.1$ & $49.2 \pm 2.4$ & $\begin{array}{l}-12.976 \\
<.001^{\&}\end{array}$ \\
\hline
\end{tabular}

* chi-square test; \# Student's t test;\& Mann-Whitney U test. Multiple comparison was adjusted with Bonferroni calculation

a. The difference was significant $(P<0.05)$ after multiple comparison correction

heterogeneous, such as bacterial or virus infection from vagina or blood. Even chronic stress could lead to it [60]. In animal research, when exposed to lipopolysaccharide (LPS), deficient vitamin D diet mice were observed with an elevation mRNA for Il-6, IFN$\gamma$, TNF- $\alpha$, classical inflammatory factors, in placenta. However, researchers haven't reached agreement on the anti-inflammatory effect of vitamin D during pregnancy.

\section{Low birth weight/SGA}

Consistent with our study, some researchers noted maternal serum vitamin D were scarcely related to SGA, while others showed that mothers with lower vitamin D levels were apt to giving birth to SGA fetuses [4]. Wang indicated that maternal VDD might be an independent risk factor for poor fetal growth, for each $1 \mathrm{ng} / \mathrm{ml} \mathrm{de-}$ crease of $25(\mathrm{OH}) \mathrm{D}$ accompanied by $19 \%$ increase of the risk of SGA [61]. This might be explained by the following mechanisms: First, maternal VDD may affect directly fetal bone metabolism. Then, as a member of steroid hormone family, vitamin $\mathrm{D}$ could interact with other hormones vital for fetal development, such as thyroid hormone [62]. The homeostasis of metabolism of nutrient in pregnancy could be modulated by vitamin $\mathrm{D}$, affecting fetal development [63].

\section{Strengths of the study}

This study with large sample size and focused on the vitamin D status of pregnant women in the first trimester in easter coastal China, Shanghai. The result highlights the deficiency of vitamin D was prevalent, not only in developed countries. We analyzed a potential correlation between vitamin D deficiency and maternal and neonatal outcomes such as preterm birth, gestational diabetes mellitus, preeclampsia, intrauterine inflammation, SGA and admission to NICU of newborns. Of the most important, we found a strong association between maternal vitamin D levels with NICU hospitalization, a meaningful indicator of long-term health of newborns and provided policy-makers with a supportive evidence to put emphasis on vitamin D supplementation in early period of pregnancy.

\section{Limitations and weaknesses of the study}

First, this study of its retrospective nature, we couldn't determine whether lower maternal vitamin D concentrations would lead to a higher risk of NICU admission rate. Secondly, some covariates such as dietary, lifestyle, sunshine exposure, clothing preferences and extra supplementation of vitamin D were not available in the study. Finally, as every coin has two sides, we didn't record the vitamin D levels of the second and third trimesters, just focused on the first trimester. It might be possible that the levels of vitamin D would have changed progressively. Further well-designed and prospective researches are necessary for clarify the causal link between maternal vitamin D status and the outcomes of mothers and newborns.

\section{Conclusion}

Maternal vitamin D deficiency $(25(\mathrm{OH}) \mathrm{D}<50 \mathrm{nmol} / \mathrm{L})$ was prevalent in easter coastal China. The incidence rate of GDM as well as preeclampsia was higher in vitamin D insufficient group while vitamin D deficiency group was liable to intrauterine infection. Furthermore, low vitamin D status in pregnant women was an independent risk factor for admission to NICU. More well-designed perspective researches are necessary to clarify the role of vitamin $\mathrm{D}$ in the early stage of pregnancy.

\section{Abbreviations}

DOHaD: Developmental Origin of Health and Disease; VDD: Vitamin D deficiency; BMI: Body Mass Index; NICU: Neonatal Intensive Care Unit; 
PTB: Preterm Birth; PROM: Preterm Rupture of Membranes ; GDM: Gestational Diabetes Mellitus; LBG: Low birth weight; VLBW: Very low birth weight;

SGA: Small for gestational age; LGA: Large for gestational age;

LPS: Lipopolysaccharide

\section{Acknowledgments}

This work was supported by a grant from National Institute of Health. The authors would like to thank Chengyu Pan for excellent statistic support and practical advises.

\section{Authors' contributions}

All the authors contributed to the work approved the final version of the manuscript. Particularly, contributions were: study design: NM, LZW, ZQQ, ZJR; Data collection: NM, WT, YTD; Data analyses and interpretation: NM, SQW; Manuscript drafting: NM; Critical revision of the manuscript: LZW, ZQQ, ZJR, NM

\section{Funding}

This work was supported by the National Key R\&D Program of China under Grant 2016YFC1000203; National Natural Science Foundation of China under Grant 81974232; Chinese Academy of Medical Sciences Research Unit under Grant 2019RU056; National Natural Science Foundation of China under Grant Sh82001646; anghai Jiao Tong University, CAMS Innovation Fund for Medical Sciences under Grant 2019-12M-5-064.

\section{Availability of data and materials}

The data used during the study are available from the corresponding author on reasonable request.

\section{Declarations}

\section{Ethics approval and consent to participate}

The study was approved by the International Peace Maternity and Child Health Hospital's committee on human research. The ethics approval number is (GKLW) 2016-21. Informed consents had been obtained from all participants or their parents (age under 16) in accordance with the Declaration of Helsinki.

\section{Consent for publication}

Not applicable.

\section{Competing interests}

The authors declare no conflict of interest.

\section{Author details}

${ }^{1}$ International Peace Maternity and Child Health Hospital, School of Medicine, Shanghai Jiao Tong University, 910\# Hengshan Road, Shanghai 20030, China. ${ }^{2}$ International Peace Maternity and Child Health Hospital of China Welfare Institution, School of Medicine, Shanghai Jiao Tong University, Shanghai, China. ${ }^{3}$ Shanghai Key Laboratory of Embryo Original Disease, Shanghai, China.

\section{Received: 23 October 2020 Accepted: 17 May 2021}

\section{Published online: 29 July 2021}

\section{References}

1. Wacker M, Holick MF. Vitamin D - effects on skeletal and extraskeletal health and the need for supplementation. Nutrients. 2013;5(1):111-48.

2. Wilson LR, Tripkovic L, Hart KH, Lanham-New SA. Vitamin D deficiency as a public health issue: using vitamin D2 or vitamin D3 in future fortification strategies. Proc Nutr Soc. 2017;76(3):392-9.

3. Palacios C, Gonzalez L. Is vitamin D deficiency a major global public health problem? J Steroid Biochem Mol Biol. 2014; 144 Pt A:138-145.

4. Aghajafari F, Nagulesapillai T, Ronksley PE, Tough SC, O'Beirne M, Rabi DM. Association between maternal serum 25-hydroxyvitamin D level and pregnancy and neonatal outcomes: systematic review and meta-analysis of observational studies. BMJ. 2013;346:f1169.

5. Dawodu A, Saadi HF, Bekdache G, Javed Y, Altaye M, Hollis BW. Randomized controlled trial $(\mathrm{RCT})$ of vitamin $\mathrm{D}$ supplementation in pregnancy in a population with endemic vitamin D deficiency. J Clin Endocrinol Metab. 2013;98(6):2337-46.
6. Diogenes MEL, Bezerra FF, Rezende EP, Taveira MF, Pinhal I, Donangelo CM. Effect of calcium plus vitamin D supplementation during pregnancy in Brazilian adolescent mothers: a randomized, placebo-controlled trial. Am J Clin Nutr. 2013:98(1):82-91.

7. Asemi Z, Tabassi Z, Heidarzadeh Z, Khorammian H, Sabihi S-S, Samimi M. Effect of calcium-vitamin D supplementation on metabolic profiles in pregnant women at risk for pre-eclampsia: a randomized placebocontrolled trial. Pak J Biol Sci. 2012;15(7):316-24.

8. Rosendahl J, Pelkonen AS, Helve O, Hauta-Alus H, Holmlund-Suila E, Valkama S, et al. High-dose vitamin D supplementation does not prevent allergic sensitization of infants. J Pediatr. 2019;209:139-145.e1.

9. McGrath J. Does 'imprinting' with low prenatal vitamin D contribute to the risk of various adult disorders? Med Hypotheses. 2001;56(3):367-71.

10. Ponsonby A-L, Lucas RM, Lewis S, Halliday J. Vitamin D status during pregnancy and aspects of offspring health. Nutrients. 2010;2(3):389-407.

11. Holick MF, Binkley NC, Bischoff-Ferrari HA, Gordon CM, Hanley DA, Heaney $\mathrm{RP}$, et al. Evaluation, treatment, and prevention of vitamin D deficiency: an Endocrine Society clinical practice guideline. J Clin Endocrinol Metab. 2011; 96(7):1911-30.

12. Christoph $P$, Challande $P$, Raio L, Surbek D. High prevalence of severe vitamin $D$ deficiency during the first trimester in pregnant women in Switzerland and its potential contributions to adverse outcomes in the pregnancy. Swiss Med Wkly. 2020;150:w20238.

13. Toko EN, Sumba OP, Daud II, Ogolla S, Majiwa M, Krisher JT, et al. Maternal vitamin D status and adverse birth outcomes in children from rural Western Kenya. Nutrients. 2016;8(12):794.

14. Zhang Q, Chen H, Wang Y, Zhang C, Tang Z, Li H, et al. Severe vitamin D deficiency in the first trimester is associated with placental inflammation in high-risk singleton pregnancy. Clin Nutr. 2019;38(4):1921-6.

15. Nimitphong H, Holick MF. Vitamin D status and sun exposure in Southeast Asia. Dermatoendocrinol. 2013;5(1):34-7.

16. Song SJ, Si S, Liu J, Chen X, Zhou L, Jia G, et al. Vitamin D status in Chinese pregnant women and their newborns in Beijing and their relationships to birth size. Public Health Nutr. 2013;16(4):687-92.

17. Xiang F, Jiang J, Li H, Yuan J, Yang R, Wang Q, et al. High prevalence of vitamin $D$ insufficiency in pregnant women working indoors and residing in Guiyang, China. J Endocrinol Invest. 2013;36(7):503-7.

18. Zhou J, Su L, Liu M, Liu Y, Cao X, Wang Z, et al. Associations between 25hydroxyvitamin D levels and pregnancy outcomes: a prospective observational study in southern China. Eur J Clin Nutr. 2014;68(8):925-30.

19. Townsend K, Evans KN, Campbell MJ, Colston KW, Adams JS, Hewison M. Biological actions of extra-renal 25-hydroxyvitamin D-1alpha-hydroxylase and implications for chemoprevention and treatment. J Steroid Biochem Mol Biol. 2005;97(1-2):103-9.

20. Anderson CM, Gillespie SL, Thiele DK, Ralph JL, Ohm JE. Effects of maternal vitamin D supplementation on the maternal and infant Epigenome. Breastfeed Med. 2018:13(5):371-80.

21. Wang TJ, Zhang F, Richards JB, Kestenbaum B, van Meurs JB, Berry D, et al. Common genetic determinants of vitamin $D$ insufficiency: a genome-wide association study. Lancet. 2010;376(9736):180-8.

22. Kennedy DA, Cooley K, Skidmore B, Fritz H, Campbell T, Seely D. Vitamin d: pharmacokinetics and safety when used in conjunction with the pharmaceutical drugs used in cancer patients: a systematic review. Cancers (Basel). 2013;5(1):255-80.

23. Ross AC. The 2011 report on dietary reference intakes for calcium and vitamin D. Public Health Nutr. 2011;14(5):938-9.

24. Lagunova Z, Porojnicu AC, Lindberg F, Hexeberg S, Moan J. The dependency of vitamin D status on body mass index, gender, age and season. Anticancer Res. 2009;29(9):3713-20.

25. Chen $\mathrm{Y}-\mathrm{H}$, Fu L, Hao J-H, Wang H, Zhang C, Tao F-B, et al. Influent factors of gestational vitamin $\mathrm{D}$ deficiency and its relation to an increased risk of preterm delivery in Chinese population. Sci Rep. 2018;8(1):3608.

26. Bodnar LM, Catov JM, Simhan HN, Holick MF, Powers RW, Roberts JM. Maternal vitamin D deficiency increases the risk of preeclampsia. J Clin Endocrinol Metab. 2007;92(9):3517-22.

27. Brannon PM. Vitamin D and adverse pregnancy outcomes: beyond bone health and growth. Proc Nutr Soc. 2012;71(2):205-12.

28. Veena SR, Gale CR, Krishnaveni GV, Kehoe SH, Srinivasan K, Fall CH. Association between maternal nutritional status in pregnancy and offspring cognitive function during childhood and adolescence; a systematic review. BMC Pregnancy Childbirth. 2016;16:220. 
29. Maia-Ceciliano TC, Dutra RR, Aguila MB, Mandarim-De-Lacerda CA. The deficiency and the supplementation of vitamin D and liver: lessons of chronic fructose-rich diet in mice. J Steroid Biochem Mol Biol. 2019;192: 105399.

30. Cooper C, Harvey NC, Bishop NJ, Kennedy S, Papageorghiou AT, Schoenmakers I, et al. Maternal gestational vitamin D supplementation and offspring bone health (MAVIDOS): a multicentre, double-blind, randomised placebo-controlled trial. Lancet Diabetes Endocrinol. 2016;4(5):393-402.

31. Liao X-P, Chipenda-Dansokho S, Lewin A, Abdelouahab N, Wei S-Q. Advanced neonatal medicine in China: a National Baseline Database. PLoS One. 2017;12(1):e0169970.

32. Rios JD, Shah PS, Beltempo M, Louis D, Mukerji A, Premji S, Shah V, Lee SK, Pechlivanoglou P. Costs of neonatal intensive care for Canadian infants with preterm birth. J Pediatr. 2021;229:161-167.e12.

33. Deindl $P$, Diemert A. From structural modalities in perinatal medicine to the frequency of preterm birth. Semin Immunopathol. 2020;42(4):377-83.

34. Liu L, Oza S, Hogan D, Perin J, Rudan I, Lawn JE, et al. Global, regional, and national causes of child mortality in 2000-13, with projections to inform post-2015 priorities: an updated systematic analysis. Lancet. 2015;385(9966): 430-40.

35. Blencowe H, Cousens S, Chou D, Oestergaard M, Say L, Moller A-B, Kinney M, Lawn J. Born too soon: the global epidemiology of 15 million preterm births. Reprod Health. 2013; 10 Suppl 1:S2.

36. Dawson-Hughes B, Heaney RP, Holick MF, Lips P, Meunier PJ, Vieth R. Estimates of optimal vitamin D status. Osteoporos Int. 2005;16(7):713-6.

37. Morley R, Carlin JB, Pasco JA, Wark JD. Maternal 25-hydroxyvitamin D and parathyroid hormone concentrations and offspring birth size. J Clin Endocrinol Metab. 2006;91(3):906-12.

38. Tabatabaei N, Auger N, Herba CM, Wei S, Allard C, Fink GD, et al. Maternal vitamin D insufficiency early in pregnancy is associated with increased risk of preterm birth in ethnic minority women in Canada. J Nutr. 2017;147(6): $1145-51$.

39. Wei S-Q, Qi H-P, Luo Z-C, Fraser WD. Maternal vitamin D status and adverse pregnancy outcomes: a systematic review and meta-analysis. J Matern Fetal Neona. 2013;26(9):889-99.

40. Flood-Nichols SK, Tinnemore D, Huang RR, Napolitano PG, Ippolito DL. Vitamin D deficiency in early pregnancy. PLoS One. 2015;10(4):e0123763.

41. Hollis BW, Wagner CL. Vitamin D supplementation during pregnancy: improvements in birth outcomes and complications through direct genomic alteration. Mol Cell Endocrinol. 2017;453:113-30.

42. Boyle VT, Thorstensen EB, Mourath D, Jones MB, McCowan LME, Kenny LC, et al. The relationship between 25 -hydroxyvitamin $D$ concentration in early pregnancy and pregnancy outcomes in a large, prospective cohort. $\mathrm{Br}$ J Nutr. 2016;116(8):1409-15.

43. Yu L, Guo Y, Ke H-J, He Y-S, Che D, Wu J-L. Vitamin D status in pregnant women in southern China and risk of preterm birth: a large-scale retrospective cohort study. Med Sci Monit. 2019:25:7755-62.

44. Rosenfeld T, Salem H, Altarescu G, Grisaru-Granovsky S, Tevet A, Birk R. Maternal-fetal vitamin D receptor polymorphisms significantly associated with preterm birth. Arch Gynecol Obstet. 2017;296(2):215-22.

45. Wahab RJ, Scholing JM, Gaillard R. Maternal early pregnancy dietary glycemic index and load, fetal growth, and the risk of adverse birth outcomes. Eur J Nutr. 2021:60(3):1301-11.

46. Tsai P-JS, Roberson E, Dye T. Gestational diabetes and macrosomia by race/ ethnicity in Hawaii. BMC Res Notes. 2013;6:395.

47. Akbari M, Moosazaheh M, Lankarani KB, Tabrizi R, Samimi M, Karamali M, et al The effects of vitamin D supplementation on glucose metabolism and lipid profiles in patients with gestational diabetes: a systematic review and metaanalysis of randomized controlled trials. Horm Metab Res. 2017:49(9):647-53.

48. Alvarez JA, Ashraf A. Role of vitamin d in insulin secretion and insulin sensitivity for glucose homeostasis. Int J Endocrinol. 2010;2010:351-85.

49. Hassan-Smith Z, Hewison M, Gittoes N. Vitamin D supplementation and prevention of type 2 diabetes. N Engl J Med. 2019;381(18):1784-5.

50. Vaidya A, Williams JS. Vitamin D and insulin sensitivity: can gene association and pharmacogenetic studies of the vitamin $D$ receptor provide clarity? Metabolism. 2012;61(6):759-61.

51. Looker AC, Gunter EW. Hypovitaminosis D in medical inpatients. N Engl J Med. 1998;339(5):344-5.

52. Davies-Tuck M, Yim C, Knight M, Hodges R, Doery JCG, Wallace E. Vitamin D testing in pregnancy: does one size fit all? Aust N Z J Obstet Gynaecol. 2015;55(2):149-55
53. Hyppönen E, Cavadino A, Williams D, Fraser A, Vereczkey A, Fraser WD, et al. Vitamin D and pre-eclampsia: original data, systematic review and metaanalysis. Ann Nutr Metab. 2013;63(4):331-40.

54. Akbari S, Khodadadi B, Ahmadi SAY, Abbaszadeh S, Shahsavar F. Association of vitamin D level and vitamin D deficiency with risk of preeclampsia: a systematic review and updated meta-analysis. Taiwan J Obstet Gynecol. 2018;57(2):241-7.

55. Ganguly A, Tamblyn JA, Finn-Sell S, Chan S-Y, Westwood M, Gupta J, Kilby MD, Gross SR, Hewison M. Vitamin D, the placenta and early pregnancy: effects on trophoblast function. J Endocrinol. 2018;236(2)R93-R103.

56. Galinsky R, Polglase GR, Hooper SB, Black MJ, Moss TJM. The consequences of chorioamnionitis: preterm birth and effects on development. J Pregnancy. 2013;2013:412831.

57. Bastek JA, Weber AL, McShea MA, Ryan ME, Elovitz MA. Prenatal inflammation is associated with adverse neonatal outcomes. Am J Obstet Gynecol. 2014; 210(5):450.e451-450.410.

58. Villamor-Martinez E, Álvarez-Fuente M, Ghazi AMT, Degraeuwe P, Zimmermann LJ, Kramer BW, et al. Association of Chorioamnionitis with Bronchopulmonary Dysplasia among Preterm Infants: a systematic review, meta-analysis, and Metaregression. JAMA Netw Open. 2019;2(11):e1914611.

59. Torchin H, Lorthe E, Goffinet F, Kayem G, Subtil D, Truffert P, et al. Histologic Chorioamnionitis and Bronchopulmonary dysplasia in preterm infants: the epidemiologic study on low gestational ages 2 cohort. J Pediatr. 2017;187.

60. Vrachnis N, Vitoratos N, lliodromiti Z, Sifakis S, Deligeoroglou E, Creatsas G. Intrauterine inflammation and preterm delivery. Ann N Y Acad Sci. 2010; 1205:118-22.

61. Wang H, Xiao Y, Zhang L, Gao Q. Maternal early pregnancy vitamin D status in relation to low birth weight and small-for-gestational-age offspring. J Steroid Biochem Mol Biol. 2018;175:146-50.

62. Mousavi SE, Amini H, Heydarpour P, Amini Chermahini F, Godderis L. Air pollution, environmental chemicals, and smoking may trigger vitamin $D$ deficiency: evidence and potential mechanisms. Environ Int. 2019;122:67-90.

63. Tamblyn JA, Hewison M, Wagner CL, Bulmer JN, Kilby MD. Immunological role of vitamin D at the maternal-fetal interface. J Endocrinol. 2015;224(3): R107-21.

64. Roth DE, Morris SK, Zlotkin S, Gernand AD, Ahmed T, Shanta SS, et al. Vitamin D supplementation in pregnancy and lactation and infant growth. N Engl J Med. 2018;379(6):535-46

65. Adami S, Viapiana O Fau - Gatti D, Gatti D Fau - Idolazzi L, Idolazzi L Fau Rossini M, Rossini M. Relationship between serum parathyroid hormone, vitamin D sufficiency, age, and calcium intake. (8756-3282 (Print)).

66. Smulian JC, Shen-Schwarz S, Vintzileos AM, Lake MF, Ananth CV. Clinical chorioamnionitis and histologic placental inflammation. Obstet Gynecol. 1999:94(6):1000-5.

67. Steel JH, O'Donoghue K, Kennea NL, Sullivan MHF, Edwards AD. Maternal origin of inflammatory leukocytes in preterm fetal membranes, shown by fluorescence in situ hybridisation. Placenta. 2005;26(8-9):672-7.

68. Olmos-Ortiz A, Avila E, Durand-Carbajal M, Díaz L. Regulation of calcitrio biosynthesis and activity: focus on gestational vitamin $D$ deficiency and adverse pregnancy outcomes. Nutrients. 2015;7(1):443-80.

\section{Publisher's Note}

Springer Nature remains neutral with regard to jurisdictional claims in published maps and institutional affiliations.

Ready to submit your research? Choose BMC and benefit from:

- fast, convenient online submission

- thorough peer review by experienced researchers in your field

- rapid publication on acceptance

- support for research data, including large and complex data types

- gold Open Access which fosters wider collaboration and increased citations

- maximum visibility for your research: over $100 \mathrm{M}$ website views per year

At BMC, research is always in progress.

Learn more biomedcentral.com/submission 